\title{
A Semi-Empirical Performance Study of Two-Hop DSRC Message Relaying at Road Intersections
}

\author{
Hieu Nguyen ${ }^{1}\left(\mathbb{D}\right.$, Md. Noor-A-Rahim ${ }^{1, *(\mathbb{D})}$, Zilong Liu $^{2}$, Dayana Jamaludin ${ }^{1}$ \\ and Yong Liang Guan ${ }^{1}$ \\ 1 Centre for Infocomm Technology (INFINITUS), Nanyang Technological University, \\ Singapore 639798, Singapore; nguyenth@ntu.edu.sg (H.N.); jdayana@ntu.edu.sg (D.J.); \\ eylguan@ntu.edu.sg (Y.L.G.) \\ 2 Institute for Communication Systems, 5G Innovation Centre, University of Surrey, Guildford GU2 7XH, UK; \\ zilong.liu@surrey.ac.uk \\ * Correspondence: narahim@ntu.edu.sg; Tel.: +65-98971930
}

Received: 20 April 2018; Accepted: 17 June 2018; Published: 18 June 2018

\begin{abstract}
This paper is focused on a vehicle-to-vehicle (V2V) communication system operating at a road intersection, where the communication links can be either line-of-sight (LOS) or non-line-of-sight (NLOS). We present a semi-empirical analysis of the packet delivery ratio of dedicated short-range communication (DSRC) safety messages for both LOS and NLOS scenarios using a commercial transceiver. In a NLOS scenario in which the reception of a safety message may be heavily blocked by concrete buildings, direct communication between the on-board units (OBUs) of vehicles through the IEEE 802.11p standard tends to be unreliable. On the basis of the semi-empirical result of safety message delivery at an intersection, we propose two relaying mechanisms (namely, simple relaying and network-coded relaying) via a road-side unit (RSU) to improve the delivery ratio of safety messages. Specifically, we designed RSU algorithms to optimize the number of relaying messages so as to maximize the message delivery ratio of the entire system in the presence of data packet collisions. Numerical results show that our proposed relaying schemes lead to a significant increase in safety message delivery rates.
\end{abstract}

Keywords: semi-empirical analysis; packet collision; IEEE 802.11p; vehicle-to-vehicle (V2V) communication

\section{Introduction}

Vehicle-to-everything (V2X) communication plays an important role in intelligent transport systems (ITSs). There are now two approaches for V2X communication: one is dedicated short-range communication (DSRC) based on the IEEE 802.11p standard, and the other is based on the LTE-V2V standard [1,2]. The latter was developed on the basis of cellular technology and helps a vehicle to communicate with different types of devices, such as pedestrians with a mobile phone, the infrastructure/network, and other vehicles. It focuses deeply on V2V communication to exchange short messages that are used in safety-related applications. In this paper, we consider the former system, which has been in trial for a long period of time and has shown advantages in V2V communication.

A vehicle equipped with a DSRC unit designed on the basis of the IEEE 802.11p standard [3] can communicate with other vehicles to exchange warning messages in order to avoid accidents and improve the traffic situation. Safety messages such as Cooperative Awareness Messages (CAMs) [4] or Basic Safety Messages (BSMs) [5] are periodically broadcasted. These messages contain a vehicle's key information (such as location, speed, heading, etc.) given by the on-board Global Navigation Satellite System (e.g., GPS) as well as other information, such as the vehicle type and brake condition. When a vehicle receives safety messages from others, a suitable visual warning may be displayed to help the 
driver to enhance the vehicle's driving safety. The capability of receiving safety messages is crucial in a dense vehicular communication network with many vehicles attempting to broadcast their individual safety messages in an uncoordinated manner. In this paper, we consider V2V communication at a road intersection, in which the $\mathrm{V} 2 \mathrm{~V}$ safety message exchange will help to prevent accidents of vehicles approaching the intersection from different directions [6]. Intersections are a challenging environment for V2V wireless communication, as the V2V communication links may be blocked by high-rise buildings and other obstructions. Measurements have shown that the strength of the received signal power reduces quickly with distance from the intersection [7-13], resulting in significant degradation of $\mathrm{V} 2 \mathrm{~V}$ transmission performance; the packet delivery ratio reduced notably at distances of 40-50 m from the intersection [10]. Moreover, the data packet collision problem tends to be more severe at intersections, as the vehicle density is usually higher at an intersection than in other places. In previous works, the dissemination of $\mathrm{V} 2 \mathrm{~V}$ safety messages was derived for the highway environment [14]. The authors in $[15,16]$ proposed relaying algorithms at intersections. However, less is understood about the performance of the entire V2V communication system, including the drop rate and delivery ratios. Moreover, no work considers practical path-loss modeling in the relaying for intersection scenarios. More precisely, existing works on packet dissemination performance at road intersections consider a theoretical path-loss model and do not take into account the measurement-based path-loss model. We note that, in [8], the authors focus on deriving the path-loss and fading channel at an intersection on the basis of measured data. No relay or network coding is considered in [8]. In [17], a relay concept for emergency vehicles is considered to send messages to local authorities. However, relaying at intersections is not discussed.

In this paper, we analyze the performance of the V2V communication system at an intersection where one vehicle tries to exchange safety messages with other vehicles approaching from different directions (including vehicles on the same road). We consider communication links that may be LOS and/or NLOS. Experimental results are presented using commercial DSRC vehicles for both LOS and NLOS scenarios. The major contributions of this work are summarized as follows:

1. We performed experiments to measure the performance of the V2V communication for LOS and NLOS scenarios. We observed that in the case of a NLOS channel for which two vehicles travel in perpendicular directions, the V2V communication link may be highly unreliable.

2. We propose two relaying strategies, namely, simple relaying and network-coded relaying, by placing a road-side unit (RSU) at the intersection center to retransmit some safety messages to improve the transmission reliability.

3. Using the proposed relaying approaches, it was found that the delivery rate is an integer function of the contention window size, which can be heuristically maximized through numerical optimization. Efficient optimization algorithms were introduced to determine the optimal fraction of messages to be retransmitted from the relay.

4. An interesting observation found in this research is that relaying more than the optimal number of safety messages cannot increase the performance but can bring about more collisions.

The remainder of this paper is organized as follows. In Section 2, along with the system description, we present the experimental results for the LOS and NLOS scenarios. The proposed relaying schemes, namely, simple relaying and network-coded relaying, are described in Section 3, and optimization procedures are provided. In Section 4, we present the simulation results on the relaying strategies along with the optimization results. Finally, we conclude in Section 5.

\section{DSRC Point-to-Point Communication Analysis}

In a vehicle, the DSRC safety message for safety applications such as BSMs (in U.S. standard) and CAMs (in European standard) is packed into the payload part of the IEEE wireless access in vehicular environments (WAVE) signal. This short message is encapsulated to a data packet (which comprises the header, training sequences, etc.) and subsequently broadcasted periodically to other vehicles. The transmitted signal experiences path loss and fading before reaching the receiver. 
Figure 1 shows an intersection at which vehicle V1 needs to communicate with other vehicles and a RSU. For two vehicles on the same street, one can say that the communication link between them is LOS because the transmitted signal can reach from one to the other through a direct transmission path. However, if they are on different streets, the radio link may be NLOS and thus tends to be unreliable. In Figure 1, the communication link between V1 and V2 (or V4) is LOS, whereas that between V1 and V3 (or V5) is NLOS. In particular, the communication link between V1 and the RSU is LOS, and because of the high position of the RSU's antenna, we may consider this vehicle-to-infrastructure (V2I) link to be more reliable than any of the V2V links (even LOS links). In what follows, we analyze the performances of V2V communication for both LOS and NLOS scenarios under the setting of Figure 1.

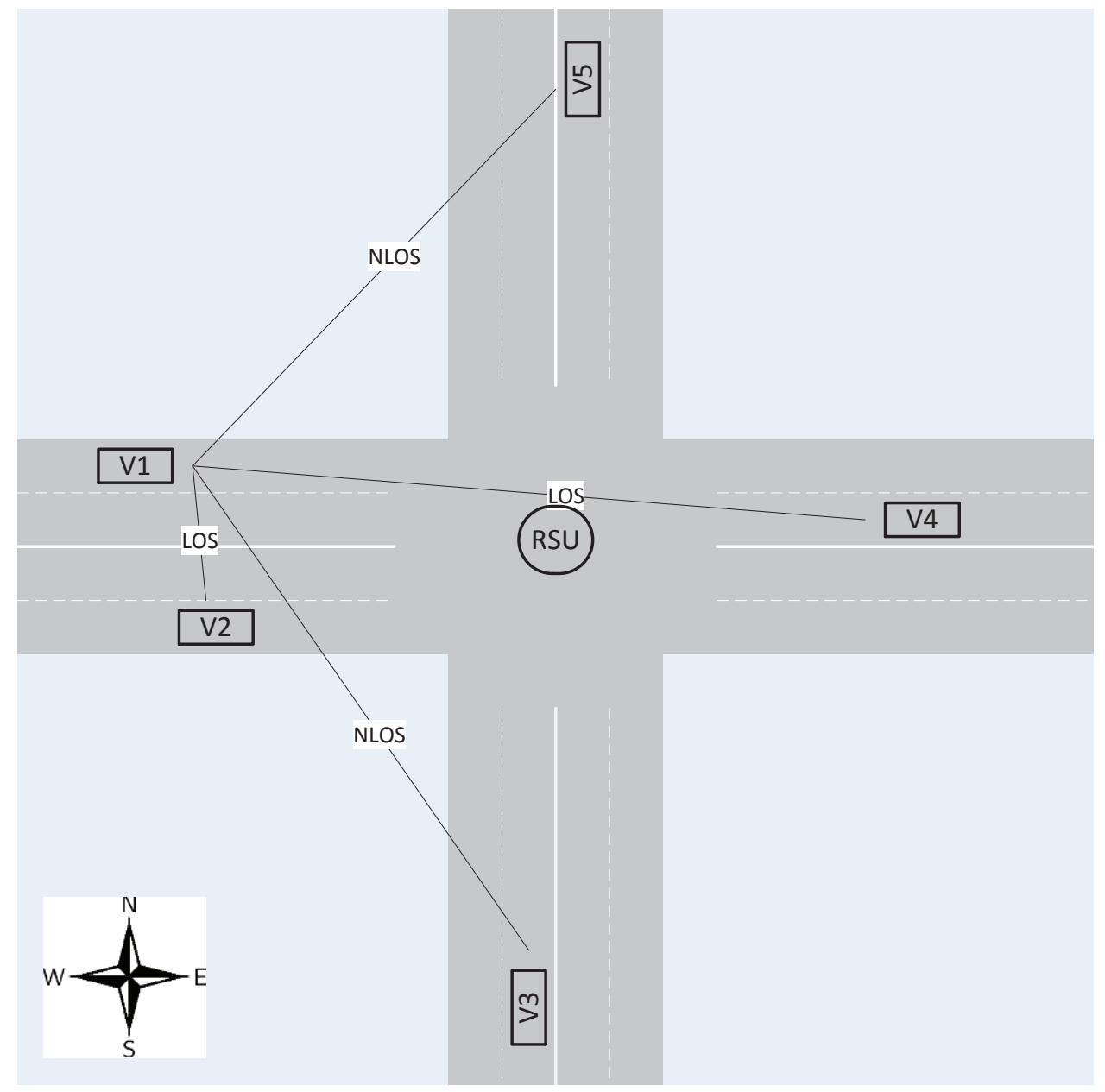

Figure 1. V2X communication at an intersection.

\subsection{Non-Line-of-Sight (NLOS) Environment}

Urban/suburban intersections are a typical environment in which every driver needs to be aware of approaching vehicles that may be blocked by high-rise buildings, trees, and so forth. In this scenario, such an intersection may be viewed as a NLOS environment in V2V communication for which the transmit antenna cannot see the receive antenna. The measurement-based model of path loss and fading at intersections has been well reported in [8], where the path loss depends on the street width, the distance of the transmitter/receiver to the intersection center (where a RSU is usually located), the distance of the transmitter to the wall, and the working frequency. The path-loss model presented in [8] is in the following page (see Equation (1)). In Equation (1), $d_{r}\left(d_{t}\right)$ denotes the distance of transmitter (Tx) (respectively, receiver $(\mathrm{Rx})$ ) from the intersection center, $w_{r}$ is the width of the $\mathrm{Rx}$ street, and $x_{t}$ is the distance of Tx from the wall; $i_{s}=1$ for a suburban environment and 0 for an urban 
environment. The path-loss exponent in NLOS is $n_{\mathrm{NLOS}}=2.69$. The fading is modeled as a Gaussian random variable, denoted by $X_{\sigma}$, with a zero mean and standard deviation of $\sigma=4.1 \mathrm{~dB}$.

$$
P L_{\mathrm{NLOS}}\left(d_{r}, d_{t}, w_{r}, x_{t}, i_{s}\right)=3.75+i_{s} 2.94+ \begin{cases}10 \log _{10}\left(\left(\frac{\frac{1}{t}^{0.957}}{\left(x_{t} w_{r}\right)^{0.81}} \frac{4 \pi d_{r}}{\lambda}\right)^{n_{\mathrm{NLOS}}}\right) & \text { if } \quad d_{r} \leq d_{b}, \\ 10 \log _{10}\left(\left(\frac{d_{t}^{0.957}}{\left(x_{t} w_{r}\right)^{0.81}} \frac{4 \pi d_{r}^{2}}{\lambda d_{b}}\right)^{n_{\mathrm{NLOS}}}\right) & \text { if } \quad d_{r}>d_{b} .\end{cases}
$$

As a result of path loss and fading, the received signal is a function of the distance between $T x / R x$ and the intersection center. Using Equation (1), as well as the fading parameter $X_{\sigma}$, the combination of path loss and fading in the form of $P L_{\mathrm{NLOS}}^{\text {fading }}\left(d_{t}, d_{r}\right)$ can be written as

$$
P L_{\mathrm{NLOS}}^{\mathrm{fading}}\left(d_{t}, d_{r}\right)=\alpha_{\mathrm{NLOS}}+10 n_{\mathrm{NLOS}} \log _{10}\left(\beta_{\mathrm{NLOS}} d_{t}^{n_{t}} d_{r}^{n_{r}}\right)+X_{\sigma},
$$

where $\alpha_{\mathrm{NLOS}}=3.75+i_{s} 2.94, n_{t}=0.957, \beta_{\mathrm{NLOS}}=\frac{1}{\left(x_{t} w_{r}\right)^{0.81}} \frac{4 \pi}{\lambda}$ or $\frac{1}{\left(x_{t} w_{r}\right)^{0.81}} \frac{4 \pi}{\lambda d_{b}}$, and $n_{r}=1$ or 2 according to $d_{r} \leq d_{b}$ or $d_{r}>d_{b}$, respectively. Assuming the transmit power is $P_{\mathrm{TX}}$, then the received signal power can be expressed as

$$
\begin{aligned}
& P_{\mathrm{RX}}\left(d_{t}, d_{r}\right) \\
= & P_{\mathrm{TX}}-P L_{\mathrm{NLOS}}^{\text {fading }}\left(d_{t}, d_{r}\right) \\
= & P_{\mathrm{TX}}-\alpha_{\mathrm{NLOS}}-10 n_{\mathrm{NLOS}} \log _{10}\left(\beta_{\mathrm{NLOS}} d_{t}^{n_{t}} d_{r}^{n_{r}}\right)-X_{\sigma} .
\end{aligned}
$$

At the receiver side, the received signal is decoded to obtain the safety message. The ability to receive the correct safety message depends heavily on the quality of the received signal. Assuming that the equalizer and channel coding can compensate for the multipath fading effect, the probability of a correct safety message being received is a function of the received signal power or the received signal-to-noise ratio (SNR), where the noise is the thermal noise of the receiver. The dependence of this probability on the received SNR varies according to the specific structure of receiver design, which can be observed for each kind of transceiver on the basis of measurements. In our work, the DSRC transceiver from Cohda Wireless was used [18] to measure the probability of correctly receiving a safety message as well as to measure the received power at the RF port (after the receive antenna). On the basis of the measured data, we applied a fitting operation to obtain the relationship between the probability of correct safety-message reception and the received power or received SNR. The measured data points and fitting functions are shown in Figure 2. For the convenience of the integral calculation in the next step, we fit the measured data with an exponential function. (The drop rate or packet error rate versus received power (or SNR) is typically an error function erf, and an erf function is usually approximated by an exponential function. This is the reason we chose to fit the measured data to an exponential function; it was near optimal. The large deviation at high received power was higher than the low received power because in that range, the error rate was low.) This was to express the probability of failure to correctly receive a safety message as a function of the received power as

$$
p_{f}\left(P_{\mathrm{RX}}\right)=a e^{-b P_{\mathrm{RX}}} .
$$

The probability of correct safety-message reception can be easily calculated as $p_{s}=1-p_{f}$. By substituting Equation (3) into Equation (4), we have

$$
\begin{aligned}
p_{f} & =a e^{-b\left(P_{\mathrm{TX}}-\alpha_{\mathrm{NLOS}}-10 n_{\mathrm{NLOS}} \log _{10}\left(\beta_{\mathrm{NLOS}} d_{t}^{n_{t}} d_{r}^{n_{r}}\right)-X_{\sigma}\right)} \\
& =a e^{-b\left(P_{\mathrm{TX}}-\alpha_{\mathrm{NLOS}}-10 n_{\mathrm{NLOS}} \beta_{\mathrm{NLOS}}\right)} d_{t}^{\frac{10 b n_{t} n_{\mathrm{NLOS}}}{\ln 10}} d_{r}^{\frac{10 b n_{r} n_{\mathrm{NLOS}}}{\ln 10}} e^{b X_{\sigma}} .
\end{aligned}
$$


The average value of this probability is given by

$$
\overline{p_{f}}=\int_{-\infty}^{+\infty} p_{f} f(X) d X
$$

where

$$
f(X)=\frac{1}{\sqrt{2 \pi \sigma^{2}}} e^{-\frac{X^{2}}{2 \sigma^{2}}}
$$

is the Gaussian probability density function of the random variable X. Substituting Equation (5) into Equation (6), we obtain the below-average failure probability of correct safety-message reception:

$$
\overline{p_{f}}=a e^{-b\left(P_{\mathrm{TX}}-\alpha_{\mathrm{NLOS}}-10 n_{\mathrm{NLOS}} \beta_{\mathrm{NLOS}}\right)} d_{t}^{\frac{10 b n_{t} n_{\mathrm{NLOS}}}{\ln 10}} d_{r}^{\frac{10 b n_{r} n_{\mathrm{NLOS}}}{\ln 10}} \frac{e^{\frac{\sigma^{2}}{2}}}{\sqrt{2 \pi \sigma^{2}}} .
$$

It then follows that the average probability of correct safety-message reception for a NLOS environment is

$$
\overline{p_{s}^{\mathrm{NLOS}}}=1-\overline{p_{f}}
$$

where $\overline{p_{f}}$ is given in Equation (7).

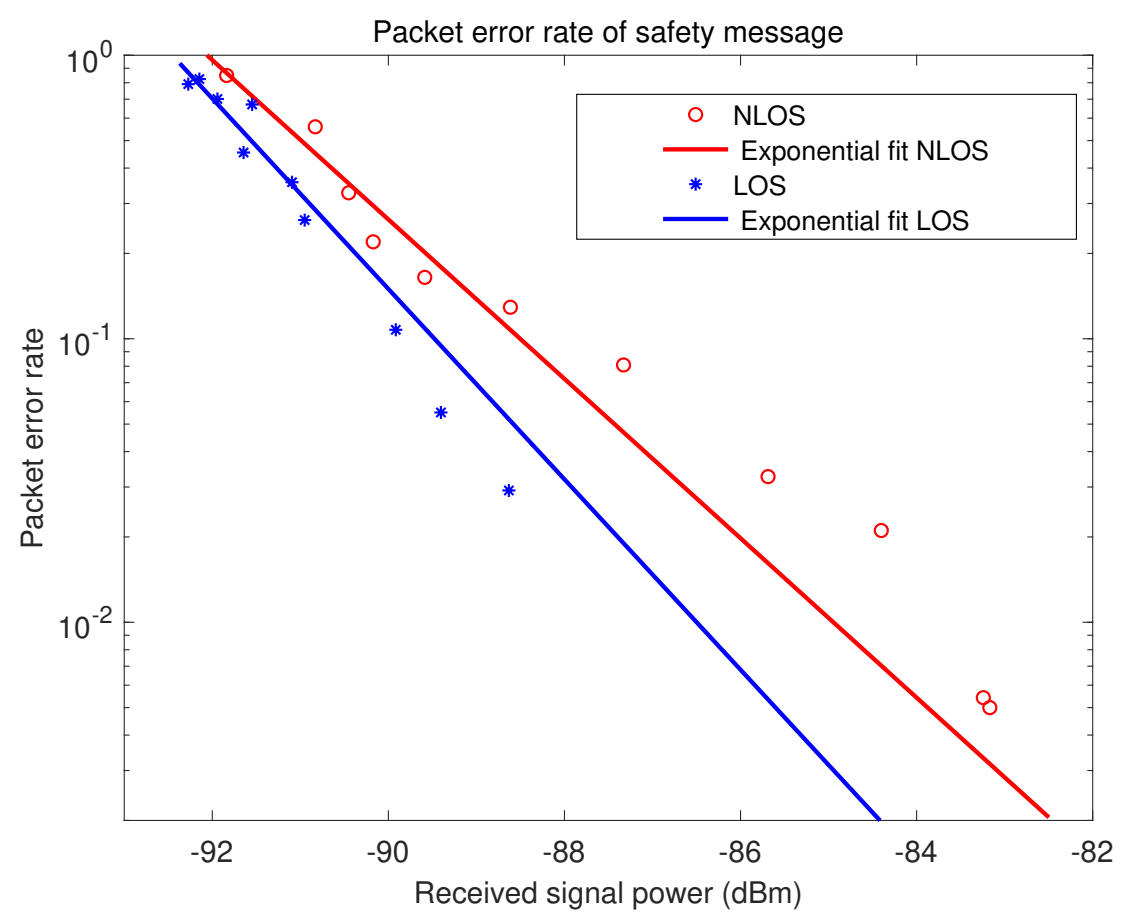

Figure 2. Packet error rate of safety messages using a commercial dedicated short-range communication (DSRC) transceiver.

\subsection{LOS Environment}

In a LOS environment, the path loss and fading are a function of the distance $d$ between Tx and $\mathrm{Rx}[19,20]$, as opposed to $d_{t}$ and $d_{r}$ (distances from the intersection center) in the NLOS scenario. By a similar analysis to that for NLOS, we can derive the average probability of correct safety-message reception for LOS environments to be

$$
\overline{p_{S}^{\mathrm{LOS}}}=1-a_{\mathrm{LOS}} \cdot e^{-b_{\mathrm{LOS}}\left(P_{\mathrm{TX}}-\alpha_{\mathrm{LOS}}-10 n_{\mathrm{LOS}} \beta_{\mathrm{LOS}}\right)} \times d^{\frac{10 b_{\mathrm{LO}} n_{\mathrm{LOS}}}{\ln 10}} \frac{e^{\frac{\sigma_{\mathrm{LOS}}^{2}}{2}}}{\sqrt{2 \pi \sigma_{\mathrm{LOS}}^{2}}} .
$$




\section{Relaying at Intersection}

At an intersection, we assume that there are $N$ vehicles each carrying an on-board unit (OBU) in the form of a DSRC transceiver and one RSU at the center of the intersection. Each OBU can communicate with other OBUs in a NLOS environment with the probability of connection given by Equation (8). Because there are $N$ vehicles, we have a total of $N(N-1)$ communication links among all OBUs. In general, it is difficult to maintain all the links with certain guarantees of reliability over a NLOS environment, particularly to satisfy the requirements of safety applications given in [17]. It is true that vehicles closer to the intersection are more dangerous and need to be made aware of first. However, in [17], the intersection safety application named "intersection collision avoidance" requires that the range of communication is from 200 to $300 \mathrm{~m}$. This means that risk is posed if communication with vehicles further away is not maintained.

The channel between a RSU and OBU is typically LOS and of high reliability; the probability of correct safety-message reception is close to 1 . In the following subsections in this paper, we present two relaying strategies using a RSU.

\subsection{Simple Relaying}

In this case, a RSU works as a relay and simply rebroadcasts the safety messages of $K$ OBUs. For each safety message, there is a field for the message ID. If other OBUs receive multiple replicated messages with the same message ID from one OBU, they will discard the replicated messages. We also assume that the RSU will rebroadcast the OBU's message before it expires, on the basis of the time-stamp field of each message. Instead of rebroadcasting $K$ randomly selected OBU messages, we propose the selection of $K$ OBU messages in the following manner. First, the RSU sorts the vehicles around the intersection according to their distances from the intersection center. This can be easily completed by a RSU (i.e., at the intersection center) because each OBU has a GPS receiver and its location is known to the RSU via its safety message. With the above information, the RSU can start to relay for the furthest vehicle first, then the second-furthest vehicle, and so on. The rationale behind this choice is that the furthest vehicle from the intersection center has the highest probability of losing the link with the other vehicles. Thus, relaying the BSMs in this manner will give rapid improvement in the the delivery ratio. To derive the expression for the delivery ratio of the proposed system, we define the following notation:

- $\quad P_{s}\left(v_{i} \rightarrow v_{j}\right)$ denotes the success probability of message delivery for a transmission from vehicle $v_{i}$ to vehicle $v_{j}$, where $i, j \in\{1, N\}$ :

- $\quad P_{S}\left(v_{i} \rightarrow v_{j}\right)=1$ if $i=j$;

- $\quad P_{S}\left(v_{i} \rightarrow v_{j}\right)$ is calculated from Equation (8) if $v_{i}$ and $v_{j}$ are NLOS;

- $\quad P_{S}\left(v_{i} \rightarrow v_{j}\right)$ is calculated from Equation (9) if $v_{i}$ and $v_{j}$ are LOS.

- $\quad P_{f}\left(v_{i} \rightarrow v_{j}\right)$ denotes the failure probability of message delivery, which can be calculated by $P_{f}\left(v_{i} \rightarrow v_{j}\right)=1-P_{s}\left(v_{i} \rightarrow v_{j}\right)$.

- $\quad P_{s}\left(v_{i} \rightarrow I\right)$ denotes the success probability of message delivery for a transmission from $v_{i}$ to the RSU.

- $\quad P_{s}\left(I_{m_{i}} \rightarrow v_{j}\right)$ denotes the success probability of message delivery for a retransmission of message $m_{i}$ (message of $v_{i}$ ) from the RSU to $v_{j}$.

- $\quad \mathbf{r}$ denotes an array of length $N$ that contains the index of the vehicles when the vehicles are sorted in descending order according to their distances from the intersection center. For example, $\mathbf{r}(\mathbf{1})$ is the index of the furthest vehicle, $\mathbf{r}(\mathbf{2})$ is that of the second furthest, and so on. 
With the above notations, we derive the delivery ratio of the safety message of the entire system as

$$
\begin{aligned}
& D_{\mathrm{SR}}(N, K)=\frac{1}{N(N-1)}\left\{\sum_{i=1}^{N} \sum_{j=1 ; j \neq i}^{N} P_{S}\left(v_{i} \rightarrow v_{j}\right)+\right. \\
& \left.\sum_{p=1}^{K} P_{S}\left(v_{\mathbf{r}(\mathbf{p})} \rightarrow I\right) \sum_{q=1}^{N} P_{S}\left(I_{m_{\mathbf{r}(\mathbf{p})}} \rightarrow v_{q}\right) P_{f}\left(v_{\mathbf{r}(\mathbf{p})} \rightarrow v_{q}\right)\right\},
\end{aligned}
$$

where the first term inside the curly bracket is associated to correct safety-message reception between OBUs that communicate directly, and the second term is responsible for the simple retransmission of $K \in\{1, N\}$ OBUs' BSMs through the RSU.

However, whenever a RSU rebroadcasts safety messages of $K$ OBUs, this will lead to increased channel traffic. According to [21,22], there may be increased collision numbers as well as a reduction in the successful delivery of data packets if more nodes in a network attempt to send data simultaneously to their shared medium. Because of this, we need to choose the number of OBUs (i.e., $K$ ) that are responsible for rebroadcasting appropriately. In particular, in the case that there are $K$ OBUs going through the relay, we may view the V2V system as having $K$ additional "ghost" OBUs and in total there being $N+K$ nodes in the network, which may deteriorate the probability of collisions. When a collision occurs, we discard the received message.

The problem of choosing an optimal value of $K$ is formulated as an integer optimization problem as follows:

$$
\begin{gathered}
\max _{K}\left\{D_{\mathrm{SR}}(N, K)-\frac{W^{N+K}-N+K}{W^{N+K}} \sum_{t=1}^{W-1}(W-t)^{N+K-1}\right\} \\
\text { s.t. } \quad 0 \leq K \leq N .
\end{gathered}
$$

The last part of the cost function is the probability of collision [21] caused by $N$ vehicles and the $K$ "ghost" vehicles in the network, where $W$ is the size of the contention window. In this paper, we consider an approximate packet collision probability model as proposed in [21]. The motivation of using this model was the availability of a closed-form expression for the collision probability, which allowed us to develop a tractable optimization scenario. However, this collision model is applicable to a saturation scenario (i.e., packets are always there to transmit for all the vehicles). Thus, the model is independent of the message rate.

$$
\begin{aligned}
& D_{\mathrm{NC}}(N, K)=\frac{1}{N(N-1)}\left\{\sum_{i=1}^{N} \sum_{j=1}^{N} P_{S}\left(v_{i} \rightarrow v_{j}\right)+\right. \\
& \sum_{p=1}^{K} P_{S}\left(v_{\mathbf{x}(\mathbf{p})} \rightarrow I\right) P_{S}\left(v_{\mathbf{y}(\mathbf{p})} \rightarrow I\right) \sum_{q=1}^{N} P_{S}\left(I_{\left.m_{\mathbf{x}(\mathbf{p})} \oplus m_{\mathbf{y}(\mathbf{p})} \rightarrow v_{q}\right)}\right. \\
& \left.\times\left[P_{S}\left(v_{\mathbf{y}(\mathbf{p})} \rightarrow v_{q}\right) P_{f}\left(v_{\mathbf{x}(\mathbf{p})} \rightarrow v_{q}\right)+P_{S}\left(v_{\mathbf{x}(\mathbf{p})} \rightarrow v_{q}\right) P_{f}\left(v_{\mathbf{y}(\mathbf{p})} \rightarrow v_{q}\right)\right]\right\}
\end{aligned}
$$

\subsection{Network-Coded Relaying}

Now we propose a network-coded relaying scheme, in which the RSU performs exclusive-OR (X-OR) operation between two vehicles' messages and then broadcasts the X-OR message. It is well known that multiple data items can be broadcast in a broadcast tick in encoded form using network coding, which results in a boost in the throughput. However, instead of performing networking coding between a pair of randomly selected vehicles, we propose the following selection and broadcasting procedure. Without loss of generality, we consider an intersection of two streets, such as the intersection shown in Figure 1, which is symmetrical in four directions. For a given street, first the RSU sorts the vehicles in that street according to their distances from the intersection center. Then the RSU broadcasts 
a network-coded message by performing X-OR between the messages of two vehicles of different streets provided that those vehicles are the furthest vehicles in their corresponding streets. After this, the RSU broadcasts a network-coded message of the two second-furthest vehicles of different streets, and so on. For simplicity, we set $N=2 \times M$, where $M$ is the number of vehicles in each street. To show the delivery ratio, we consider the following notation:

- $\quad m_{i} \oplus m_{k}$ represents a message resulting from the X-OR between the message $m_{i}$ (message of $v_{i}$ ) and the message $m_{k}$ (message of $v_{k}$ ).

- $\quad P_{S}\left(I_{m_{i} \oplus m_{k}} \rightarrow v_{j}\right)$ denotes the success probability of message delivery for the transmission of message $m_{i} \oplus m_{k}$ from the intersection relay to $v_{j}$ provided that $v_{i}$ and $v_{k}$ are in two different streets.

- $\quad \mathbf{x}$ and $\mathbf{y}$ are arrays of length $M$ that contain the indexes of the vehicles in streets WE and NS, respectively, when the vehicles are sorted in descending order according to their distances from the intersection center. For example, $\mathbf{x}(\mathbf{1})$ is the index of the furthest vehicle in street 1 , and $\mathbf{y}(\mathbf{2})$ is the index of the second-furthest vehicle in street 2 .

With the above notation and proposed relaying strategy, the relay first broadcasts $m_{\mathbf{x}(\mathbf{1})} \oplus m_{\mathbf{y}(\mathbf{1})}$, then $m_{\mathbf{x}(\mathbf{2})} \oplus m_{\mathbf{y}(\mathbf{2})}, m_{\mathbf{x}(\mathbf{3})} \oplus m_{\mathbf{y}(\mathbf{3})}$, and so on. We let $K \in\{0,1, \ldots, M\}$ be the number of broadcasts performed by the RSU. For the proposed network-coded relaying scheme, the delivery ratio of the entire system is given in the previous page (see Equation (12)). The second line inside the curly bracket is associated to the proposed network-coded broadcasting performed by the RSU. Because of the collision effect, the problem of choosing an optimal value of $K$ is formulated as an integer optimization problem as follows:

$$
\begin{gathered}
\max _{K}\left\{D_{\mathrm{NC}}(N, K)-\frac{W^{N+K}-N+K}{W^{N+K}} \sum_{t=1}^{W-1}(W-t)^{N+K-1}\right\} \\
\text { s.t. } \quad 0 \leq K \leq N .
\end{gathered}
$$

\section{Numerical Results and Discussions}

\subsection{Simulation Parameters}

A simulation was performed in MATLAB to verify the effectiveness of the proposed relaying algorithm at an intersection. We assumed there were $N$ vehicles at an intersection of two streets each with six lanes. This intersection was symmetrical in four directions, as shown in Figure 1. The area of interest was a square of $400 \times 400 \mathrm{~m}^{2}$. The other parameters are listed in Table 1 . We performed the simulation while distributing the vehicles around the intersection randomly. The delivery-ratio performance was then calculated on the basis of the distance from the transmitter and the receiver to the intersection center. The whole process was iterated over 1000 times, and then the average delivery ratio was calculated.

Table 1. Simulation parameters.

\begin{tabular}{cc}
\hline Parameter & Value \\
\hline Channel & $178(5.89 \mathrm{GHz})$ \\
Transmit power & $23 \mathrm{dBm}$ \\
Antenna gain & $3 \mathrm{~dB}$ \\
Number of lanes per street & 6 \\
Lane width & $3 \mathrm{~m}$ \\
Rx street width & $12 \mathrm{~m}$ \\
Distance of Tx from wall & $5 \mathrm{~m}$ \\
Critical distance $d_{b}$ & $100 \mathrm{~m}$ \\
\hline
\end{tabular}




\subsection{Simulation Results}

At first, we investigated the safety-message delivery ratio in the NLOS scenario without the RSU relaying, which is a difficult environment compared to the LOS scneario. Figure 3 shows the delivery ratio when the distance of $\mathrm{Tx} / \mathrm{Rx}$ to the intersection center increased from 10 to $200 \mathrm{~m}$. We measured packet error rate versus received power in LOS and NLOS (intersection corner blocked by building) by a Cohda/Wireless OBU. (In the Cohda/Wireless OBU and RSU, the safety message, such as a BSM, can be transmitted/received full time (no switching from $\mathrm{CCH}$ to $\mathrm{SCH}$ ) in one dedicated RF port. The switching mode between $\mathrm{CCH}$ and $\mathrm{SCH}$ is for non-safety applications (such as TCP/UDP) in another RF port. This paper considers the safety application mode.) The path-loss model in [8] has been validated from real-world measurements. Both the experiments and the path-loss model were used to draw the heat map. In the experiments, we measured the packet error performance of the OBU, which is the packet error rate versus the received power. On the other hand, we used the path-loss model in [8] to calculate the received power for a transmitter/receiver pair at a location in the region of interest. The combination of the above factors was used to predict the PDR for any location in the heat map. In the area close to the center, the delivery ratio was close to 1 . However, it decreased very fast when $\mathrm{Tx} / \mathrm{Rx}$ moved away from the intersection center. The distance from $\mathrm{Tx} / \mathrm{Rx}$ to the intersection center when the delivery ratio was as high as 1 was $50 \mathrm{~m}$. When the distance of $\mathrm{Tx} / \mathrm{Rx}$ from the intersection center kept increasing, the delivery ratio dropped quickly to zero. For two NLOS vehicles traveling at $70 \mathrm{~km} / \mathrm{h}$ towards the same intersection, $50 \mathrm{~m}$ indicates $2 \mathrm{~s}$ of reaction time, which is very short and hence dangerous. In this case, the relaying for vehicles in a NLOS environment is necessary.

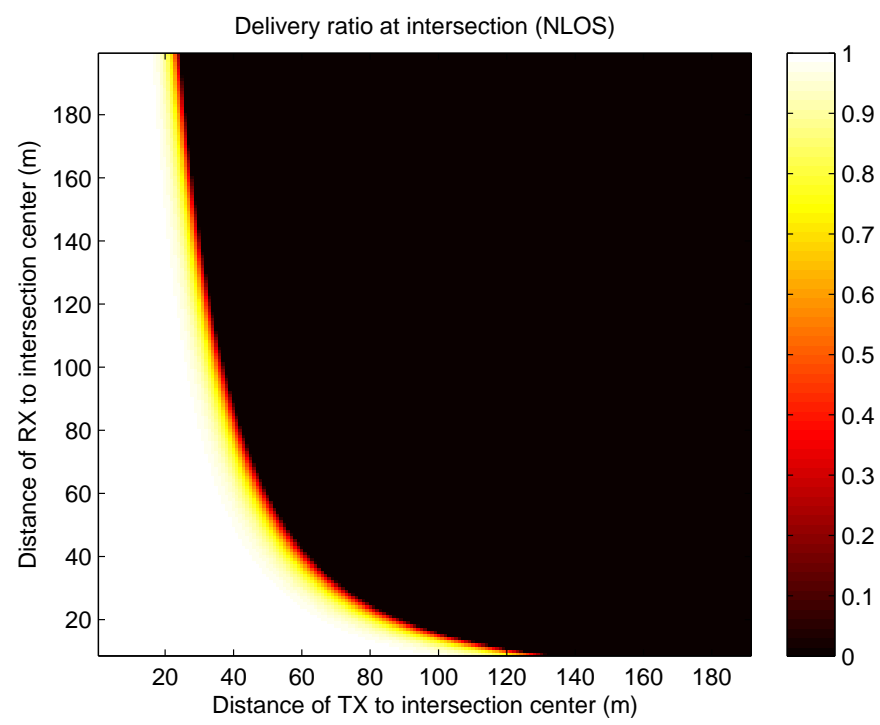

Figure 3. Delivery ratio of safety message at intersection on basis of Equation (8) without road-side unit (RSU) relaying.

The performances of the proposed schemes are shown in Figure 4 in terms of the delivery ratio versus the number of transmissions from the RSU. In the simulation, collision-free communication was assumed. A significant improvement (about 19.4\% improvement over 15 transmissions from the RSU) was observed using the network-coded relaying over the simple relaying scheme. As expected, when the number of transmissions from the RSU increased, the delivery ratio increased and reached the maximum of 1 . The reason is that the unreliable NLOS V2V links are replaced by two reliable LOS V2I links through the RSU. In Figure 4, we also show the delivery ratio when the RSU performed transmissions of messages of randomly selected vehicles. From the performance comparison, we observe that our proposed schemes completely outperformed the randomly selected schemes. Another example of the performances for a system with 60 vehicles is illustrated in Figure 5. 
Figure 6 shows the probability of collision in the V2X communication network when the number of transmissions from the RSU increased. The results for the probability of collision for contention window sizes of 32, 64, and 128 are presented. We note that in the IEEE 802.11p standard, the maximum contention window size can be 1024 [23]. When the contention window size is small, the waiting time for each OBU node before attempting to transmit is short, and the probability of collision in the network is high. When the contention window size increases, the waiting time is longer, and correspondingly, the probability of collision decreases. However, if the waiting time is too long, the safety message may expire, because the transmission rate is $10 \mathrm{~Hz}$ and the transmission time is only $100 \mathrm{~ms}$. The probability of collision for a system with 60 vehicles is illustrated in Figure 7.

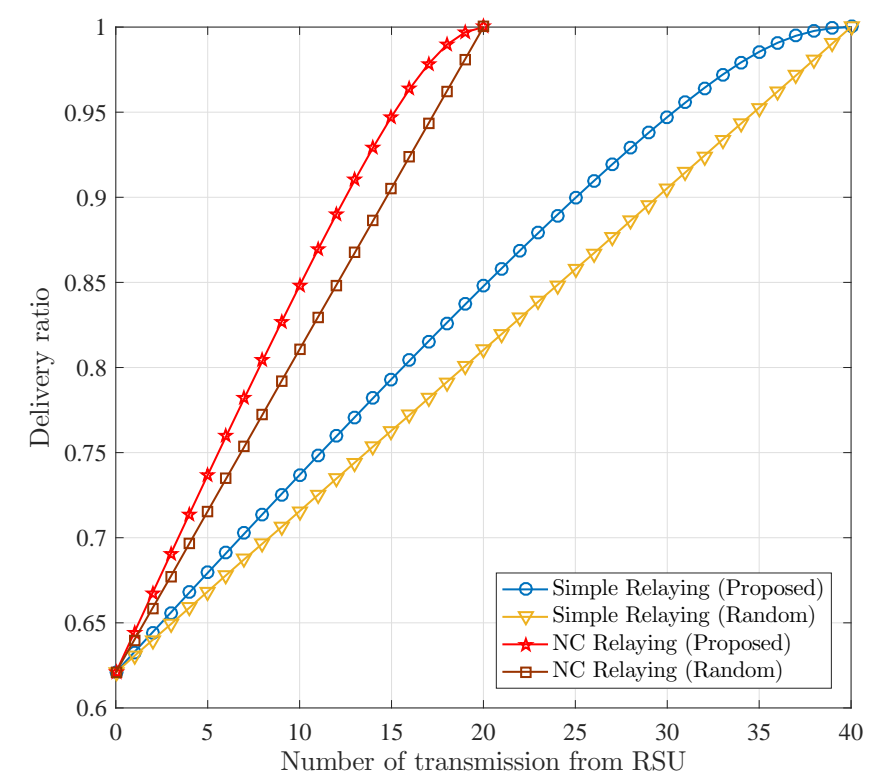

Figure 4. Delivery ratio of safety message with relaying, assuming collision-free communication (for $\mathrm{N}=40$ ).

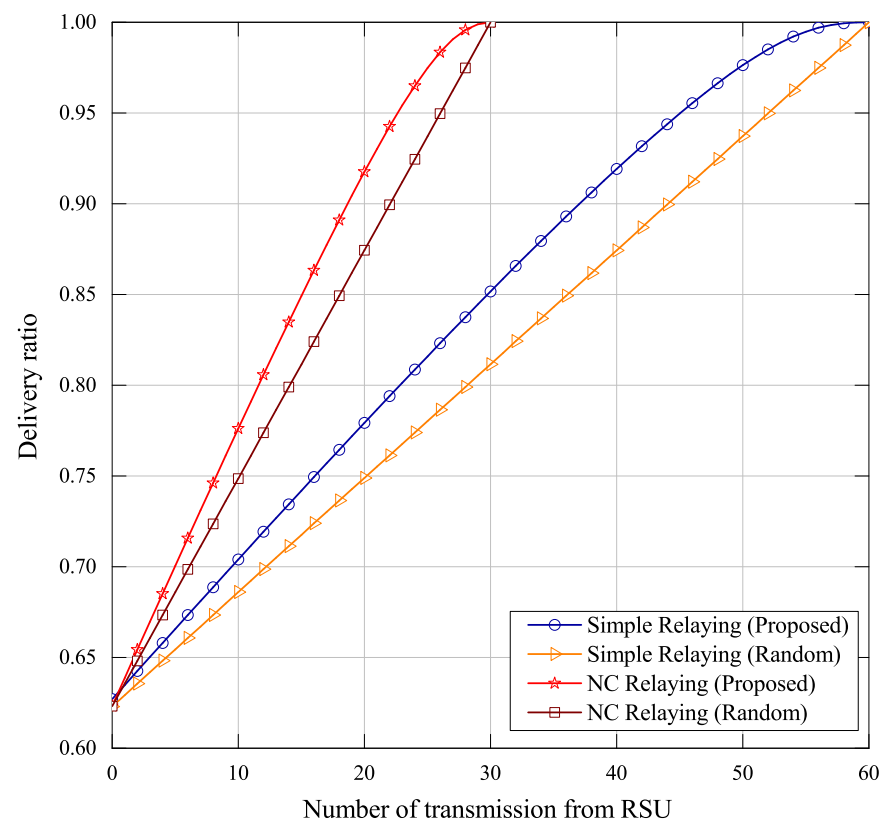

Figure 5. Delivery ratio of safety message with relaying, assuming collision-free communication (for $\mathrm{N}=60$ ). 


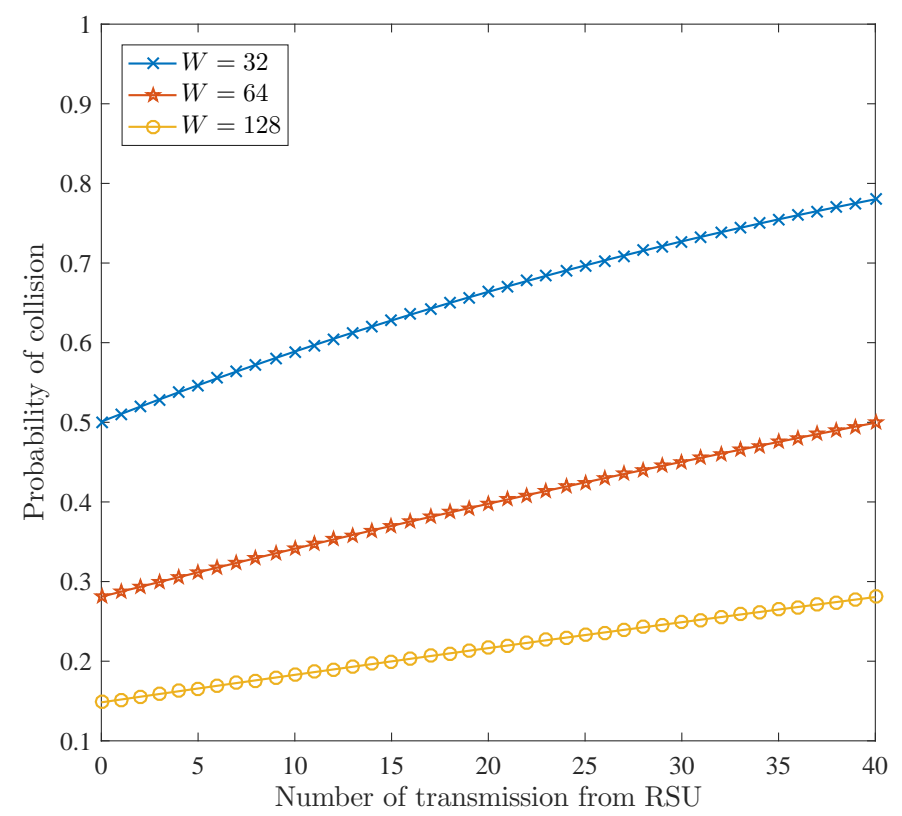

Figure 6. Probability of collision with different sizes of contention window (for $\mathrm{N}=40$ ).

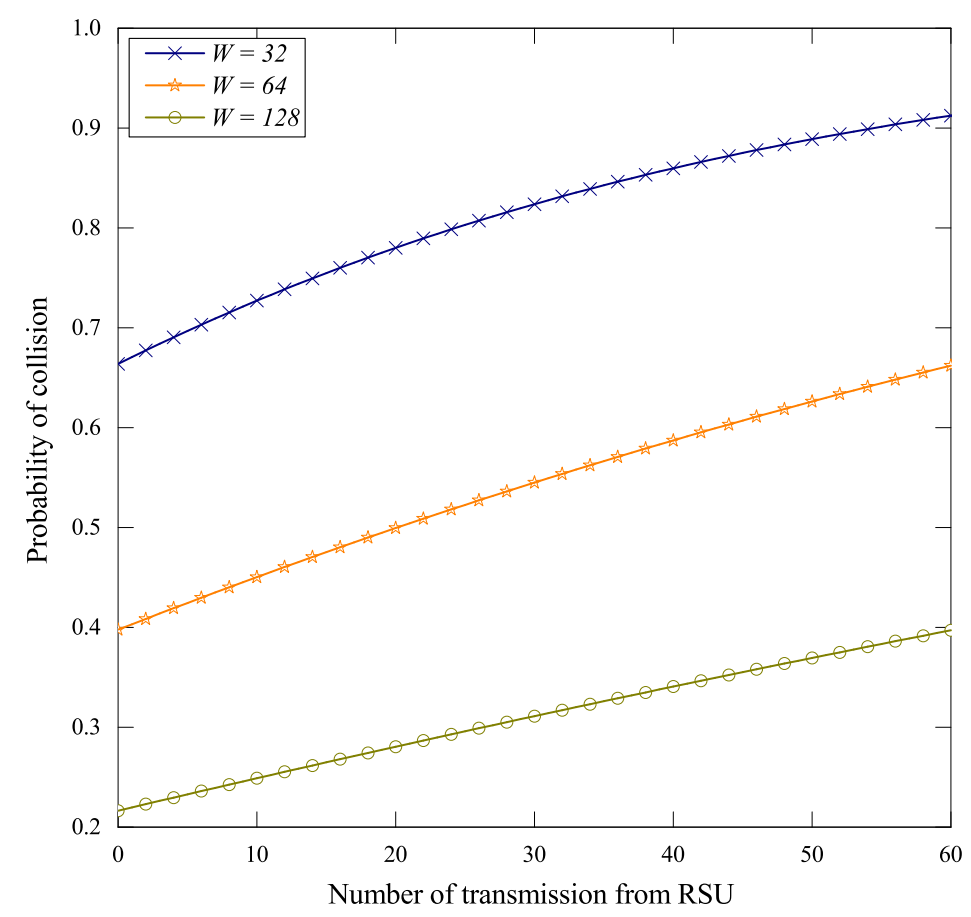

Figure 7. Probability of collision with different sizes of contention window (for $N=60$ ).

The characteristics of the cost functions obtained from Equations (11) and (13) are shown in Figure 8 , where the performances are shown in the presence of collisions with different $(K, W)$. For both proposed schemes, the cost function increased as the number of transmissions from the RSU increased. After it reached the peak value, it started to decrease as $K$ kept increasing. This was because the benefit of relaying was more than compensated for by the penalty of additional collisions caused by retransmission. For different $W$, the optimal number of relayed messages achieved by the search algorithm is depicted in the figures. 


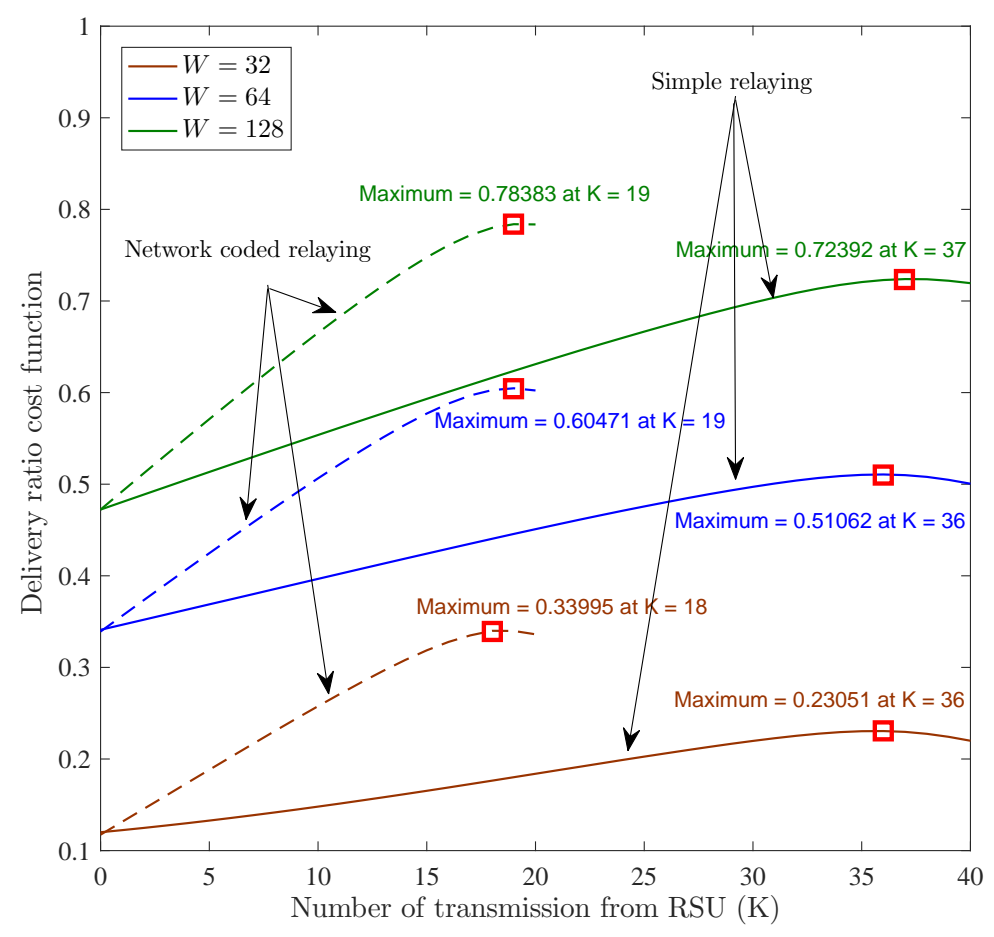

Figure 8. Delivery-ratio cost function with simple relaying and network-coded relaying schemes considering packet collision in the network. The solid lines correspond to simple relaying scheme, while the dotted lines represent network-coded relaying scheme.

\section{Conclusions}

In this paper, we analyze the performance of safety-message delivery between vehicles at intersections under LOS or NLOS communication scenarios. For both scenarios, we present experimental results on the drop rate of safety messages, for which the experiments were conducted using a commercial DSRC transceiver. In order to improve the transmission reliability in the NLOS communication scenario, we propose two relaying schemes (simple relaying and network-coded relaying) using a RSU at the intersection as a relay node to retransmit some safety messages. For the proposed relaying schemes, the delivery ratios of the entire system are presented. We also propose a search algorithm for the optimal number of relayed messages. Relaying more than the optimal value cannot increase the delivery rate but brings about more collisions. In future, it will be interesting to incorporate a more sophisticated collision model and DSRC channel utilization into our proposed schemes.

Author Contributions: H.N. and D.J. performed the experiments and data collection. M.N.-A.-R. and Z.L. were involved in theoretical investigation and optimization. Y.L.G. supervised the experimental and theoretical works.

Funding: This work was supported by the NTU-NXP Intelligent Transport System Test-Bed Living Lab Fund S15-1105-RF-LLF from the Economic Development Board, Singapore.

Conflicts of Interest: The authors declare no conflict of interest.

\section{References}

1. Seo, H.; Lee, K.D.; Yasukawa, S.; Peng, Y.; Sartori, P. LTE evolution for vehicle-to-everything services. IEEE Commun. Mag. 2016, 54, 22-28. [CrossRef]

2. Bazzi, A.; Masini, B.M.; Zanella, A.; Thibault, I. On the Performance of IEEE 802.11p and LTE-V2V for the Cooperative Awareness of Connected Vehicles. IEEE Trans. Veh. Technol. 2017, 66, 10419-10432. [CrossRef] 
3. IEEE. IEEE Standard for Information Technology_Local and Metropolitan Area Networks_Specific RequirementsPart 11: Wireless LAN Medium Access Control (MAC) and Physical Layer (PHY) Specifications Amendment 6: Wireless Access in Vehicular Environments; IEEE: New Jersey, NJ, USA, 2010; pp. 1-51. [CrossRef]

4. European Telecommunications Standards Institute (ETSI). Intelligent Transport Systems (ITS); Vehicular Communications; Basic Set of Applications; Part 2: Specification of Cooperative Awareness Basic Service; Technical Report, EN 302 637-2 V1.3.0 Draft; ETSI: Sophia Antipolis, France, 2013.

5. DSRC Committee. SAE 2735 Dedicated Short Range Communications (DSRC) Message Set Dictionary; Technical Report; SAE International: Warrendale, PA, USA, 2009.

6. Joerer, S.; Segata, M.; Bloessl, B.; Cigno, R.L.; Sommer, C.; Dressler, F. A Vehicular Networking Perspective on Estimating Vehicle Collision Probability at Intersections. IEEE Trans. Veh. Technol. 2014, 63, 1802-1812. [CrossRef]

7. Ahmed-Zaid, F.; Bai, F.; Bai, S.; Basnayake, C.; Bellur, B.; Brovold, S.; Brown, G.; Caminiti, L.; Cunningham, D.; Elzein, H.; et al. Vehicle Safety Communications Applications (VSC-A) Final Report: Appendix Volume 2 Communications and Positioning; Technical Report, DOT HS 811 492C; U.S. Department of Transportation: Washington, DC, USA, 2011.

8. Mangel, T.; Klemp, O.; Hartenstein, H. $5.9 \mathrm{GHz}$ inter-vehicle communication at intersections a validated non-line-of-sight path-loss and fading model. EURASIP J. Wirel. Commun. Netw. 2011, 2011, 182. [CrossRef]

9. Abbas, T.; Thiel, A.; Zemen, T.; Mecklenbrauker, C.F.; Tufvesson, F. Validation of a non-line-of-sight path-loss model for V2V communications at street intersections. In Proceedings of the 13th International Conference on ITS Telecommunications (ITST), Tampere, Finland, 5-7 November 2013; pp. 198-203. [CrossRef]

10. Sepulcre, M.; Gozalvez, J.; Hernandez, J. Cooperative vehicle-to-vehicle active safety testing under challenging conditions. Transp. Res. Part C Emerg. Technol. 2013, 26, 233-255. [CrossRef]

11. Bazzi, A.; Zanella, A.; Masini, B.M. A distributed virtual traffic light algorithm exploiting short range V2V communications. Ad Hoc Netw. 2016, 49, 42-57. [CrossRef]

12. Ali, G.G.M.N.; Noor-A-Rahim, M.; Chong, P.H.J.; Guan, Y.L. Analysis and improvement of reliability through coding for safety message broadcasting in urban vehicular networks. IEEE Trans. Veh. Technol. 2018. [CrossRef]

13. Noor-A-Rahim, M.; Ali, G.G.M.N.; Nguyen, H.; Guan, Y.L. Performance Analysis of IEEE 802.11p Safety Message Broadcast With and Without Relaying at Road Intersection. IEEE Access 2018, 6, 23786-23799. [CrossRef]

14. Fallah, Y.P.; Huang, C.L.; Sengupta, R.; Krishnan, H. Analysis of Information Dissemination in Vehicular Ad-Hoc Networks With Application to Cooperative Vehicle Safety Systems. IEEE Trans. Veh. Technol. 2011, 60, 233-247. [CrossRef]

15. Trien, L.T.; Yamao, Y. An investigation of network coding relay in ITS V2V communication at intersections. In Proceedings of the IEEE International Conference on Network Infrastructure and Digital Content, Beijing, China, 19-21 September 2014; pp. 297-301. [CrossRef]

16. Xu, Z.; Bernado, L.; Gan, M.; Hofer, M.; Abbas, T.; Shivaldova, V.; Mahler, K.; Smely, D.; Zemen, T. Relaying for IEEE 802.11p at road intersection using a vehicular non-stationary channel model. In Proceedings of the IEEE 6th International Symposium on Wireless Vehicular Communications (WiVeC), Vancouver, BC, Canada, 14-15 September 2014; pp. 1-6. [CrossRef]

17. CAMP Vehicle Safety Communications Consortium. Vehicle Safety Communications Project Task 3 Final Report: Identify Intelligent Vehicle Safety Applications Enabled by DSRC; Technical Report, DOT HS 809 859; National Highway Traffic Safety Administration, US Department of Transportation: Washington, DC, USA, 2005.

18. CohdaWieless. MK5 V2X on Board Unit. Technical Report. Available online: http:/ / www.cohdawireless. com/solutions/hardware/mk5-obu/ (accessed on 15 June 2018).

19. Cheng, L.; Henty, B.E.; Stancil, D.D.; Bai, F.; Mudalige, P. Mobile Vehicle-to-Vehicle Narrow-Band Channel Measurement and Characterization of the $5.9 \mathrm{GHz}$ Dedicated Short Range Communication (DSRC) Frequency Band. IEEE J. Sel. Areas Commun. 2007, 25, 1501-1516. [CrossRef]

20. Abbas, T.; Sjöberg, K.; Karedal, J.; Tufvesson, F. A measurement based shadow fading model for vehicle-to-vehicle network simulations. Int. J. Antennas Propag. 2015, 2015. [CrossRef]

21. Eichler, S. Performance Evaluation of the IEEE 802.11p WAVE Communication Standard. In Proceedings of the IEEE Vehicular Technology Conference, Baltimore, MD, USA, 30 September-3 October 2007; pp. 2199-2203. [CrossRef] 
22. Campolo, C.; Molinaro, A.; Vinel, A.; Zhang, Y. Modeling Event-Driven Safety Messages Delivery in IEEE 802.11p/WAVE Vehicular Networks. IEEE Commun. Lett. 2013, 17, 2392-2395. [CrossRef]

23. Han, C.; Dianati, M.; Tafazolli, R.; Kernchen, R. Throughput Analysis of the IEEE 802.11p Enhanced Distributed Channel Access Function in Vehicular Environment. In Proceedings of the Vehicular Technology Conference Fall (VTC 2010-Fall), Ottawa, ON, Canada, 6-9 September 2010; pp. 1-5. [CrossRef]

(C) 2018 by the authors. Licensee MDPI, Basel, Switzerland. This article is an open access article distributed under the terms and conditions of the Creative Commons Attribution (CC BY) license (http:/ / creativecommons.org/licenses/by/4.0/). 